International Journal of Biology, Pharmacy and Allied Seiences (IJBPAS) 'A Bridge Betusen caboratory and QRado'

WwW.ijbpas.com

\title{
CROSS CULTURAL ADAPTATION, TRANSLATION AND VALIDATION OF GUJARATI VERSION OF SHORT FORM - INTERNATIONAL PHYSICAL ACTIVITY QUESTIONNAIRE
}

\section{GAURAVGIRI GOSAI ${ }^{1}$, YAGNIK DAVE ${ }^{2 *}$, JAYESHBHAI PARMAR ${ }^{3}$}

1: PhD scholar, Faculty of Physiotherapy, Marwadi University, Rajkot

2: Assistant Professor, Faculty of Physiotherapy, Marwadi University, Rajkot

3: Principal, KK Sheth College of Physiotherapy Rajkot

*Corresponding Author: Yagnik Dave: E Mail: yagnikdave93@gmail.com

Received $3^{\text {rd }}$ Oct. 2021; Revised $11^{\text {th }}$ Nov. 2021; Accepted $20^{\text {th }}$ Dec. 2021; Available online $25^{\text {th }}$ Jan. $^{2022}$

https://doi.org/10.31032/iibpas/2022/11.1.2063

\section{ABSTRACT}

Introduction: Short form - International Physical Activity Questionnaire (SF-IPAQ) is a reliable and valid tool that helps to measure physical activity of an individual.

Aim: Cross Cultural Adaptation, Translation and validation of the Gujarati version of SF-IPAQ to be used in clinical research on Gujarati population.

Study Settings and design: Cross-sectional study conducted at Marwadi University, Rajkot, Gujarat, India.

Materials and Method: SF-IPAQ was translated into Gujarati from English using forwardbackward-forward method. For face \& content validity of Gujarati version group consensus method was used. Each question was examined by group of experts in field of Cardiopulmonary Physiotherapy. Each question was analyzed for content, meaning, wording, format, ease of administration \& scoring. Each question was scored by expert group as either accepted, rejected or accepted with modification. Procedure was continued until $80 \%$ of consensus for all items. Concurrent validity was found by comparing the English version of IPAQ with Gujarati version of IPAQ. 
Statistical Analysis: Spearman's correlation coefficients were used to assess the strength of association between the measures of both the versions

Results: In validation process of Gujarati version of SF-IPAQ all 7 questions were accepted. Total physical activity was significantly and highly correlated $(\rho=0.79)$ between the SF-IPAQ Gujarati and the original English version of SF-IPAQ. The significantly positive correlation found between the SF-IPAQ Gujarati and the original English version of SF-IPAQ $(\rho=0.88)$.

Conclusion: Gujarati version of Short form of International Physical Activity Questionnaire has a good validity to be used in Gujarati Population to evaluate level of physical activity.

\section{Keyword: Gujarati Version, Validation, Short Form - International Physical Activity Questionnaire}

\section{INTRODUCTION}

Physical inactivity is a very important public health issue. Sedentary lifestyles are associated with increased obesity, type 2 diabetes and cardiovascular diseases, hence the promotion of active lifestyles is an important public health priority to reduce the diseases and disorders.

There are various tools available to measure physical activity such as self reported questionnaires, physical activity diaries, accelerometers, motion sensors, heart rate monitors, etc. International physical activity questionnaire is one of such questionnaire. It is a reliable and valid tool that is used for measuring physical activity. (1)

But now a day short form of International physical activity questionnaire (SF-IPAQ) commonly used in clinical and research for the evaluation of individuals. It is available in self reported format as well as telephonic interview. The long and short forms of the questionnaire are available. (2)

SF-IPAQ use for the youth 15 years of age and older. Total 7 items included and it is Open-ended questions surrounding individuals' last 7-day recall of physical activity. It is also Self-reported for evaluation only paper-pencil requires. This tool is Available in: English and many other languages. SF-IPAQ is reliable and valid tool to assess the level of physical activity.

The SF-IPAQ was found to have acceptable criterion validity and is a useful instrument. Adewale L Oyeyemi suggest in their study, the Hausa IPAQ-SF has acceptable concurrent validity and test-retest reliability for vigorous-intensity activity, walking, sitting and total physical activity.(3)

Culturally adapting the SF-IPAQ, rather than developing a new physical activity 
questionnaire, is economical and it may facilitate future comparisons among populations.

\section{MATERIALS AND METHODOLOGY}

This is a cross sectional study conducted at Marwadi University, Rajkot, Gujarat, India. Short Form - International Physical Activity Questionnaire was translated into Gujarati from English using forward-backwardforward method using the instructions given in the Short Form - International Physical Activity Questionnaire manual.

Two independent bilinguals have translated the questions into Gujarati, and subsequently the preliminary version was again back translated into English following careful cultural adaptation. Then a third bilingual translator provided a final version. For validation process, participants are experts $(n=5)$ in the field of Physiotherapy.

Consensus is defined as agreement with a question by at least $80 \%$ of participant.
Characteristic of group consensus method is selection of expert participants. Each professional was contacted personally by primary author separately for their expert opinion in first step of validation. (4)

Each item was analysed by professionals for content, meaning, wording, format, ease of administration and scoring. Each item was scored as accepted, rejected or accepted with modification. Procedure was continued until $80 \%$ of consensus for all items.

The original English version of Short Form International Physical Activity Questionnaire and newly formed Short Form International Physical Activity Questionnaire were conceptually equivalent but some questions were culturally different. But it adheres to the sense of the original version. After this 3phase process, one final scale/tool was developed. (Fig-1) 


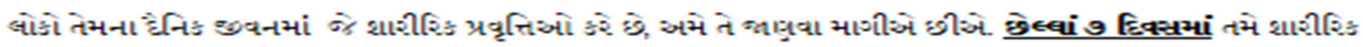

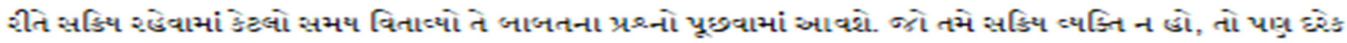

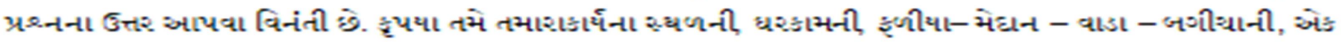

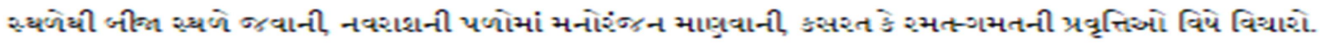

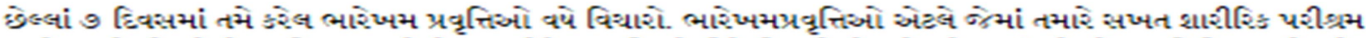

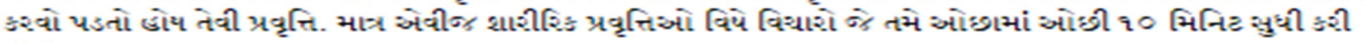
હોઈ.

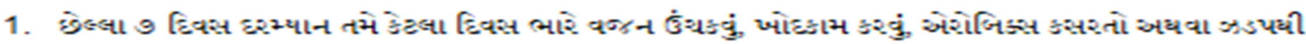
સાઈકલ ચલાવવાનેડ્વી ભારેખમ પ્રવૃત્તિઓ છી છે?

અર્વાડિયામાં દિવસ

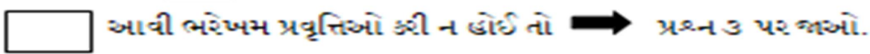

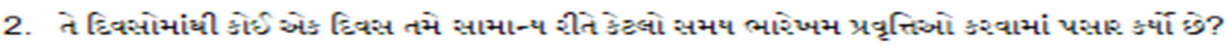

Еિવસ્યમા ક્લાક

Еિવ્सમાં મિનિટ

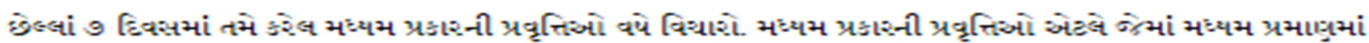

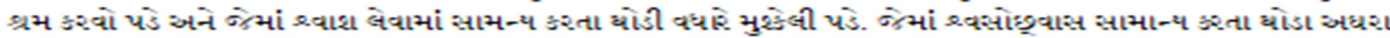

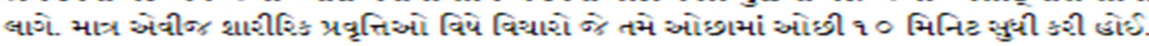

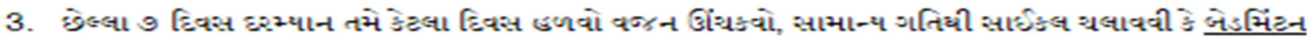

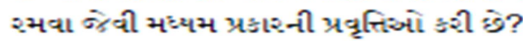

અહવાડિયામાં हिवस्स.

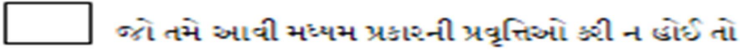

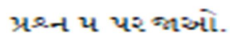

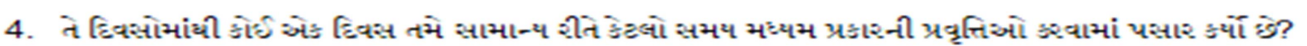
દિવસમાં કसा

Еિવસसभાં મિનિટ

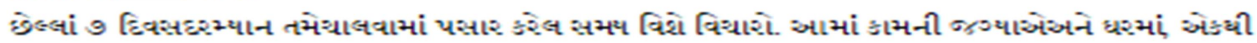

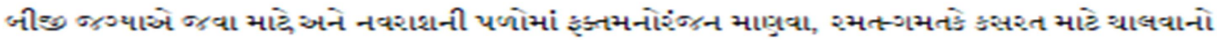
સમાવેશ ધાથ છે.

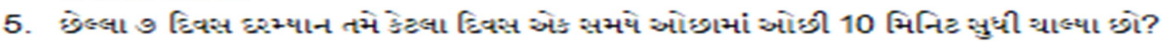
અદવાડિયામાં हिव:स.

$\square$ નો તમે ચાલ્થા ન હો, તો

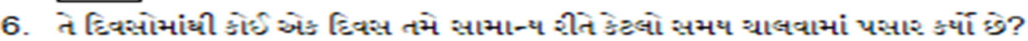

દિવેની કલાङ

દિવસલની મિનિટ

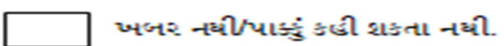

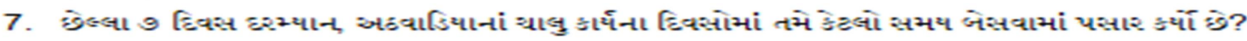
દિવ્સની __ ક્લા દિવસની —િિનિટ

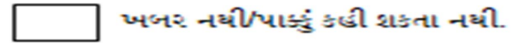

આ પ્રશ્નોત્તશીનો ઝંત અર્હી છે, ભાગ લેવા માટે તમાશો આભાર.

Fig-1: Gujarati translated Short Form International Physical Activity Questionnaire

For concurrent validity, both the versions

(English and Gujarati) of Short Form International Physical Activity Questionnaire were given to 32 subjects. All the subjects had knowledge of both languages. A simple random technique (flip of coin) was used for 
determining the order of administration of both questionnaires.

Subjects whose coin turned head first completed English version first and subjects whose coin turned tail first completed Gujarati version first. An interval of 1 hour was kept between administrations of two questionnaires.(4)

\section{RESULTS}

\section{Face \& content validity:}

In validation process of Gujarati version of SF-IPAQ was done about translation and application of certain questions in questionnaire. In question no 4 doubles tennis replaced with sports activity and approval got from the committee.

Concurrent validity:

For correlation of total short form physical activity score of Gujarati version with total physical activity score of English version Spearman's correlation test was used. Spearman correlation coefficients ranged from moderate $(\rho=0.79)$ to high $(\rho=0.88)$, indicating good concurrent validity for the Short- Form International Physical Activity Questionnaire.

Table 1: Represents the demographic details of study participants

\begin{tabular}{|c|c|c|}
\hline & Age (Mean \pm SD) & Body Mass Index (Mean \pm SD) \\
\hline Female (n=13) & $23.8 \pm 0.5$ & $24.16 \pm 1.2$ \\
\hline Male (n=19) & $22.6 \pm 0.9$ & $21.32 \pm 2.6$ \\
\hline
\end{tabular}

\section{DISCUSSION}

There is much emphasis today on using standardized and validated research instruments as these enable comparison of results both within and across countries. A patient reported outcome is an umbrella term that covers a whole range of potential types of measurement; it is used specifically to refer to measures that quantify a patient's level of physical activities in terms of outcomes reported by patients. (5)

This is the first study to validate Gujarati version of Short Form - International Physical Activity Questionnaire. The present study examined the face and content and concurrent validity of the Gujarati long, selfadministered, last 7 days version of the Short Form - International Physical Activity Questionnaire.

The results of this study indicate that the validity indices of Gujarati version of Short Form - International Physical Activity Questionnaire are similar to English version of Short Form - International Physical Activity Questionnaire. Gujarati version of Short Form - International Physical Activity Questionnaire has acceptable properties for 
assessing Physical Activities in healthy adults.

For face and content validity, none of the questions were rejected by the panel of experts. Total 7 questions were accepted with translation into Gujarati. Only 1 questions required discussion among experts and were accepted with some modification.

Craig et al has studied on reliability and validity of IPAQ among 12 countries they found IPAQ questionnaires produced repeatable data Spearman's clustered around 0.8 , with comparable data from short and long forms. Criterion validity had a median of about 0.60 , which was comparable to most other self-report validation studies. The "usual week" and "last 7 day" reference periods performed similarly, and the reliability of telephone administration was similar to the self-administered mode. (1) In the present study moderate to high correlation was observed between answers of SF-IPAQ English and those of SF-IPAQ Gujarati, with Spearman's correlation coefficients ranging from 0.70 to 0.98 .

Future recommendations criterion validity can be done by comparing with any gold standard instruments for measuring physical activities. It can be carried out on larger population with various age groups.

\section{CONCLUSION}

Gujarati version of Short form of International Physical Activity Questionnaire has a good validity to be used in Gujarati Population to evaluate level of physical activity.

\section{Conflict of Interest: None}

Source of Funding: None

Acknowledgement: The authors would like to thank Dr. Ashish Kakkad, Dr. Hemang Jani, Dr. Phalak Buch, Dr. Rahul chhatlani, and Dr. Amit Navani for providing their valuable inputs in the study.

\section{REFERENCES}

[1] Craig CL et al International physical activity questionnaire: 12-country reliability and validity. Med Sci Sports Exerc. 2003 Aug;35(8):138195.

[2] Rathod SR, Vyas N, Sorani DM. Validation of Gujarati version of international physical activity questionnaire. Int J Health Sci Res. 2018; 8(1):122-128.

[3] Van Dinh Tran et al, Validity of the International Physical Activity Questionnaire- Short Form for Application in Asian Countries: A Study in Vietnam; Evaluation \& the Health Professions; 2018;(2): 1-12. DOI: $10.1177 / 0163278718819708$ 
[4] S Campbell et al, Consensus methods in prescribing research ; Journal of Clinical Pharmacy and Therapeutics ; 2001;26(1):5-14

[5] Dave Y, Sorani D, Patel M. Cross cultural adaptation, translation, validation and reliability analysis of
Gujarati version of hypertension quality of life questionnaire (MINICHAL). Int J Health Sci Res. 2021; 11(10):10-16. DOI: https://doi.org/

10.52403/ijhsr.20211003. 